\title{
As perspectivas dos direitos humanos sobre aborto e saúde pública no Brasil: uma revisão narrativa
}

\author{
Human rights perspectives on abortion and public health in Brazil: a narrative review \\ Perspectivas de derechos humanos sobre el aborto y la salud pública en Brasil: una \\ revisión narrativa
}

Dayanna da Silva Carvalho Faria1*, Lara Stefhany Oliveira Silva ${ }^{1}$, Lorena Leão Gomes Leahy ${ }^{1}$, Maria Paula Colombini Ferreira Viana ${ }^{1}$, Luciano de Oliveira Souza Tourinho' ${ }^{1}$, Fernanda Luiza Andrade de Azevedo'.

\section{RESUMO}

Objetivo: Discutir sobre o aborto e a saúde pública no Brasil, sob a perspectiva dos direitos humanos. Revisão Bibliográfica: $O$ abortamento é um grave problema de saúde pública principalmente em países que estão em desenvolvimento, como o Brasil. $\mathrm{O}$ aborto clandestino e inseguro representa uma das principais causas de mortalidade do Brasil. Partindo dessa verdade, é notório que o sistema de saúde brasileiro não possui uma infraestrutura adequada para se realizar o aborto. Além disso, a maioria dos médicos não são de acordo a essa prática e por vezes se esquivam de realizar tal procedimento, levando a consequências notórias para a saúde da mulher como a escolha de realizar um aborto ilegal que é responsável pela quarta causa de óbito materno no Brasil. Considerações finais: Assim, essa prática traz diversas consequências físicas e psicológicas, principalmente se realizado de forma insegura. No Brasil, a ilegalidade não impede que o aborto ocorra, estando principalmente relacionado à desigualdade social e afirmando-o como um problema de saúde pública de ordem global.

Palavras-chave: Aborto, Saúde Pública, Direitos Humanos.

\begin{abstract}
Objective: Discuss abortion and public health in Brazil from the perspective of human rights. Review Bibliography: Abortion is a serious public health problem, especially in developing countries, such as Brazil. Clandestine and unsafe abortion represents one of the main causes of mortality in Brazil. Based on this truth, it is clear that the Brazilian health system does not have an adequate infrastructure to carry out abortions. In addition, most physicians do not agree with this practice and sometimes shy away from performing such a procedure, leading to notorious consequences for women's health, such as choosing to perform an illegal abortion, which is responsible for the fourth cause of maternal death, in Brazil. Final considerations: Thus, this practice brings several physical and psychological consequences, especially if performed in an unsafe way. In Brazil, illegality does not prevent abortion from taking place, being mainly related to social inequality and affirming it as a global public health problem.
\end{abstract}

Key words: Abortion, Public Health, Human Rights.

\section{RESUMEN}

Objetivo: Discutir el aborto y la salud pública en Brasil desde la perspectiva de los derechos humanos. Revisión bibliográfica: El aborto es un grave problema de salud pública, especialmente en países en desarrollo, como Brasil. El aborto clandestino e inseguro representa una de las principales causas de mortalidad en Brasil. Con base en esta verdad, es evidente que el sistema de salud brasileño no cuenta con

${ }^{1}$ Faculdade Santo Agostinho de Itabuna (FASAI), Itabuna - BA. *E-mail: dayanna.faria@outlook.com

SUBMETIDO EM: 11/2021

ACEITO EM: 11/2021

PUBLICADO EM: 12/2021 
una infraestructura adecuada para realizar abortos. Além disso, a maioria dos médicos não são de acordo a essa prática e por vezes se esquivam de realizar tal procedimento, levando a consequências notórias para a saúde da mulher como a escolha de realizar um aborto ilegal que é responsável pela quarta causa de óbito materno en Brasil. Consideraciones finales: Por lo tanto, esta práctica trae varias consecuencias físicas y psicológicas, especialmente si se realiza de manera insegura. En Brasil, la ilegalidad no impide que se produzca el aborto, estando principalmente relacionada con la desigualdad social y afirmándola como un problema de salud pública global.

Palabras clave: Aborto, Salud Pública, Derechos Humanos.

\section{INTRODUÇÃO}

O aborto é considerado um grande problema de saúde pública no Brasil. Apesar da mulher ter alcançado voz e espaço em diversos âmbitos, o seu direito à saúde é por vezes negligenciado. Partindo dessa verdade, é importante entender que apesar da instauração de políticas públicas, campanhas e manifestações em prol do bem-estar da mulher, ela não ocupa uma posição reservada a ela (MARVÁN ML, et al., 2018).

A saúde da mulher no Brasil foi introduzida através de políticas nacionais no início do século XX, entretanto, no início as medidas eram voltadas à gravidez e parto. Com isso, as pessoas começaram a entender a necessidade de abordar sobre a temática aborto buscando informar toda a população em relação a essa problemática e expandir os direitos à saúde da mulher em todas as perspectivas. Entende-se que 0 Brasil é o país com uma das legislações penais mais restritas e rigorosas em relação ao aborto fazendo-se necessário entender a sua relação com as possíveis consequências. Devido a isso, nota-se a ineficácia do Estado em garantir o acesso à saúde pelas mulheres (CORRÊA MSM, et al., 2017).

A gravidez é sem dúvida um momento de transição, adaptação e mudança na vida de uma mulher, sendo assim, torna-se necessário meios que auxiliem essa gestante durante todo esse processo. A gravidez indesejada é a uma das principais causas de aborto induzido, portanto é necessárias medidas voltadas para o Planejamento Família, para que a gravides seja algo desejado pela família. Esse planejamento tem como objetivo, ser um acompanhamento de forma continua para a família, com isso levando a um menor número de gravidezes indesejadas e consequentemente um menor número de abortos ilegais e menor mortalidade materna (SANTOS KSS, et al., 2019).

A magnitude do aborto no Brasil é estimada em 1.054.242 abortamentos que foram induzidos em 2005. Diante desse contexto, é notório a necessidade do estudo e conhecimento da forma que as mulheres cometem esse ato de abortar para que medidas rápidas sejam tomadas ao recebê-la em um ambiente hospitalar. Analisar os efeitos da clandestinidade, os custos, as consequências e os danos é a égide dessa adversidade (CARDOSO BB, et al., 2020).

Assim, este constructo teve como objetivo discutir sobre o aborto e saúde pública no Brasil, sob a perspectiva dos direitos humanos.

\section{REVISÃO BIBLIOGRÁFICA}

O termo aborto demonstra um entendimento relacionado a interrupção da gravidez de forma voluntária, ocorrendo o falecimento do produto da concepção. Entretanto, o aborto também pode ser chamado de abortamento, que talvez seja o termo mais adequado para a ação realizada que tem como resultado o aborto (AIRES FD e CARVALHO GM, 2019).

O abortamento é um grave problema de saúde pública principalmente em países que estão em desenvolvimento, como o Brasil. Sua abordagem envolve um conjunto complexo de fatores sociais, culturais, religiosos, morais e legais. Para grande parte da população feminina, o aborto é decorrente do não planejamento reprodutivo, relacionado principalmente acerca da falta de informação sobre anticoncepção, dificuldades de acesso aos métodos, falhas no uso e a ausência da integralização do cuidado pelos serviços de saúde. É valido salientar, que para outras mulheres a interrupção do ato de gestar é decorrente da imposição pelos seus parceiros ou de situações de estupro (LACERDA E, et al., 2018). 
Os aspectos culturais, religiosos e legais inibem as mulheres a declarem seus abortamentos, o que acaba dificultando calcular a sua magnitude. Mesmo diante essa dificuldade, sabe-se que o aborto é praticado vastamente, com o uso de diversos meios, o qual pode ser induzido pela própria mulher ou realizado por profissionais em condições inseguras gerando consequências nocivas à saúde, podendo levar até mesmo a morte (ESSY DB e JUSTO FS, 2019).

É válido salientar o conceito do aborto principalmente do ponto de vista médico, aborto é a interrupção da gravidez antes do período perinatal, definido pela Organização Mundial da Saúde (OMS) até 22a semana completas, ou com um feto de até 500 gramas, ou de $16,5 \mathrm{~cm}$, quer dizer, antes de atingida a viabilidade. Classifica-se o aborto como precoce quando ocorre até a 12 semanas da gravidez respondendo a $80 \%$ dos abortamentos, e como tardio quando se dá entre as 13 e 22 semanas (MENEZES G, et al., 2020).

O aborto é dividido em duas espécies: espontâneo e provocado. O aborto espontâneo ou natural ocorre sem intervenção externa, portanto não consiste em um crime. Ele é determinado por fatores intrínsecos como por doenças maternas, paternas ou por anormalidades do embrião ou feto, sendo a complicação mais frequente no primeiro trimestre, cerca de $62 \%$ após a nidação (CORREIA LL, et al., 2018).

Já o aborto provocado é à interrupção da gravidez causada por uma intervenção externa e intencional, portanto um crime caso não esteja prevista em lei. Atualmente, no Brasil, existe um grande número de casos de abortamento, em torno de um milhão, isso acaba gerando um custo para o Sistema Único de Saúde (SUS), devido as consequências do aborto que pode levar a complicações como infecção que pode ser resultado dos instrumentos utilizados, como introdução de sondas, agulhas, laminárias e soluções variadas (PAES SC, et al., 2021).

Existem também outras espécies que são o aborto acidental que não configura crime sendo decorrente de traumatismo ou acidente. O outro tipo é o aborto social ou econômico que é aquele em que o nascimento agravaria a crise financeira social, sendo que nesta modalidade haverá crime, pois não é admitido no ordenamento jurídico brasileiro (CORREIA LL, et al., 2018; BELIN LL e RIZZOTTO CC, 2021).

O aborto pode ser de diversas formas como: aborto provocado (autoaborto), no qual a própria mulher aplica os meios ou manobras abortivas em si mesma, porém é possível a participação de um terceiro, na hipótese em que este apenas induz, instiga ou auxilia, de maneira secundária, a gestante a provocar o aborto. O aborto consentido (duas figuras próprias) a mulher apenas consente na prática abortiva, mas a execução material do crime é realizada por terceira pessoa. O aborto consensual, é preciso que a gestante tenha capacidade em consentir o ato para que este seja válido. Por sim, o aborto sem consentimento da gestante é a forma mais grave do delito do aborto, no qual não é necessário que haja anuência expresso da gestante, basta apenas o emprego de meios abortivos por terceiro sem o seu conhecimento (PAES SC, et al., 2021).

\section{Legislações sobre 0 aborto}

Em todo o mundo, a taxa estimada de aborto de 2010 a 2014 em mulheres entre 14 e 15 anos foi de 0,35\%. A taxa em países ricos em recursos foi de $0,27 \%$ e em países com recursos limitados foi de $0,37 \%$. A taxa mais alta foi no Caribe (65 por 1000), e a taxa mais baixa foi na América do Norte (17 por 1000) e no Norte ou Europa Ocidental (18 por 1000). Estima-se que 25 por cento das gravidezes em todo o mundo terminaram em aborto induzido. Da mesma forma, nos Estados Unidos, cerca de uma em cada quatro mulheres terá um aborto durante sua vida reprodutiva (RODRÍGUEZ-RUÍZ AF, et al., 2021).

Partindo desses dados, alguns países que legalizaram o aborto por escolha da gestante o permitem sob o argumento de que as mulheres que o praticam ilegalmente tem altas taxas de mortalidade, invocam também a laicidade do Estado e a ideia de que até a $20^{\underline{a}}$ semana de gestação, o feto não possui viabilidade de viver extra útero, logo ele pertence ao corpo da mulher dentro desse prazo, devendo, portanto, prevalecer à soberania feminina para decidir o que fazer com o seu próprio corpo (MELO BC, 2019).

Alguns países, como Cuba, Uruguai, legalizaram o aborto sob qualquer hipótese. A institucionalização do aborto voluntário em Cuba surgiu em meados dos anos 60 , onde a mulher pode interromper a gravidez sem restrições até a $10^{\mathrm{a}}$ semana de gestação, e em casos de estupro, até a vigésima segunda. No México, em 
2007, o Distrito Federal, onde está localizada a capital do país, aprovou uma lei que permite a interrupção legal da gravidez mediante solicitação da própria mulher até a $12^{\underline{a}}$ semana de gravidez. No restante do país, porém, o aborto permanece legalmente restrito (AGUIAR BHK, et al., 2018).

O Uruguai se tornou o segundo país da América Latina a descriminalizar o aborto em qualquer hipótese, com a aprovação da Lei 18.987 de interrupção voluntária da gravidez, lançada no Sistema Nacional de Saúde Integrada em janeiro de 2013 até a décima segunda semana, em casos de estupro, o limite vai até a décima quarta semana (GALLI B, 2020).

Na França, o aborto é permitido, tanto nos casos de dificuldades socioeconômicas como em fetos que são diagnosticados com anormalidades. Além disso, todos os abortos realizados são registrados. Em relação a Alemanha, o aborto pode ser realizado em situações sociais, sendo que não existe um prazo limite para a realização do abortamento (AGUIAR BHK, et al., 2018).

Em Portugal, a legislação que alterava o Código Penal de Portugal foi aprovada em abril de 2007, permitindo que as mulheres poderiam ter acesso ao aborto até a decima semana de gestação, além disso, podendo ser realizado só após passar por psicólogos e o por um período de reflexão (VALENZUELA MT, et al., 2017).

No Canadá, as mulheres podem interromper uma gravidez voluntariamente por qualquer motivo, durante qualquer momento dos nove meses de gravidez. Na Itália, o aborto pode ser realizado até 3 meses. Nos Estados Unidos, o aborto é legal desde 1973, o país vem sofrendo alguns riscos derivados do endurecimento das leis de aborto. Diminuição do limite de semanas de gestação para a realização do aborto, que de forma geral é em torno de doze a dezesseis semanas e passaria para seis a oito semanas, outro fator importante a ser levado em consideração são as rígidas exigências para funcionamento de uma clínica de aborto (BANDEIRA JC, 2018).

Alguns países, legalizaram o aborto sob algumas condições. No Chile, o aborto era completamente proibido até o ano de 2017, marcado pela flexibilização da legislação que agora permite o procedimento em três casos: perigo para a vida da mãe, inviabilidade do feto e estupro. No Brasil, o aborto praticado por médico é permitido nos seguintes casos: se não há outro meio de salvar a vida da gestante; se a gravidez é resultado de estupro e tem o consentimento da gestante ou, quando incapaz, de seu representante legal. Além disso, também é permitido o aborto quando for comprovado que o feto é anencefálico. Esse último caso, foi aprovado em 2012 pelo Supremo Tribunal Federal (FONSECA SC, et al., 2020).

Segundo o Código Penal no Art.128 não se punirá o aborto praticado pelo médico quando: não existir outro meio de salvar a vida da gestante, ou em caso de gravidez resultante de um estupro, no qual o ato deverá ser precedido do consentimento da gestante, ou quando incapaz, de um representante legal. Ademais, no julgamento da arguição de descumprimento de preceito fundamental o Supremo Tribunal Federal (STF) declarou inconstitucional a interpretação segundo, a qual a interrupção da gravidez de feto anencéfalo, decisão que busca garantir a liberdade sexual e reprodutiva da mulher, na medida em que permite a interrupção do ato de gestar, endossando os direitos fundamentais à saúde, à dignidade, à liberdade sexual e reprodutiva, bem como a autodeterminação das mulheres (RUIBAL A, 2020).

O Código Penal Brasileiro pune o aborto através do Art. 124, auto aborto ou consentido que outrem lhe provoque com a detenção, de um a três anos. O Art. 125 traz que aborto praticado sem o consentimento da gestante tem a pena de reclusão, de três a dez anos. O Art. 126 traz que o aborto praticado com o consentimento da gestante, pena de reclusão, de um a quatro anos; sendo que o artigo 127 descreve a forma qualificada do mencionado delito (POLETTO L e FERREIRA JBS, 2020).

\section{Direito à saúde da gestante}

A mulher, no decorrer da gestação encontra-se em estado de extrema sensibilidade/vulnerabilidade, motivo pelo qual torna-se necessário que, os profissionais de saúde e os seus familiares auxiliem com a prestação de cuidados especiais. A gestante possui o direito de saúde assegurado. É vedado pela Lei $n$. 9263, de 1996, que o Sistema Único de Saúde deve garantir a mãe, atenção integral, assistência a concepção 
e contracepção, atendimento pré-natal, parto, puerpério e ao neonato. Dessa forma, no que se refere a gestante que realizou o aborto de forma necessária ou criminal, têm dificuldade de procurar ajuda médica pois tem receio do tratamento e preconceito por parte dos profissionais de saúde (SOUZA NF, et al., 2020).

$\mathrm{O}$ aborto clandestino e inseguro representa uma das principais causas de mortalidade do Brasil. Partindo dessa verdade, é notório que o sistema de saúde brasileiro não possui uma infraestrutura adequada para se realizar o aborto. Além disso, a maioria dos médicos não são de acordo a essa prática e por vezes se esquivam de realizar tal procedimento, levando a consequências notórias para a saúde da mulher como a escolha de realizar um aborto ilegal que é responsável pela quarta causa de óbito materno no Brasil (FERRARI W e PERES S, 2020).

No Brasil, configura-se o aborto como um problema de saúde pública, devido a magnitude e persistência. Segundo estimativas da Organização Mundial da Saúde (OMS), cerca de 55 milhões de abortamentos ocorreram no mundo entre 2010 e 1014, sendo a maioria abortos ilegais e sem segurança. Assim, as leis dos países que proíbem o aborto em qualquer circunstância podem colaborar para esse número tão alto de abortos. Além disso, mesmo com leis tão rígidas em alguns países, o aborto ilegal ainda ocorre, principalmente nas classes sociais menos favorecidas, com isso afirmando o aborto como um problema de ordem global (CARDOSO BB, et al., 2020).

Em relação aos Estados do Brasil, o Nordeste é o Estado que menos diminuiu os casos de aborto. Esse fator pode estar relacionado por ser uns dos locais mais pobres do Brasil e que recebem menos assistência à saúde. Outra premissa importante são as opiniões culturalmente influenciadas de médicos nordestinos que possuem importante papel nas causas de mortes (CORREIA LL, et al., 2018).

\section{Consequências sobre 0 aborto}

Sabe-se que as complicações físicas do abortamento são: tetania, sepse, perfurações uterina, restos placentários, infertilidade e inflamações uterinas. Além disso, com o avanço gestacional a chance de agravamento aumenta. Tais complicações podem afetar a vida reprodutiva futura da mulher de forma a causar esterilidade, senéquias uterinas e inflamações. Além dos riscos de gravidez ectópica, baixo peso ao nascer e abortamentos espontâneos. O grande desafio é a prevenção desses resultados prejudiciais à saúde da mulher, principalmente residentes em comunidades pobres com poucos serviços médicos disponíveis (CISNE $\mathrm{M}$, et al., 2018).

Grande parte das literaturas mostrou que quando a Idade Gestacional (IG) é menor que 12 semanas pode ter gerado um tipo de complicação mais leve, em contrapartida, os abortamentos realizados com período gestacional mais avançado que resultariam em piores prognósticos. A maioria das mulheres observadas fizeram o uso de misoprostol. Os estudos científicos têm debatido sobre a chance do acesso a este fármaco (mesmo que não seja permitido) possa reduzir a mortalidade (ESTEVES MF, et al., 2021).

Além dos perigos físicos os danos psicológicos mais recorrentes da situação de abortamento consistem em: perda da fé, sentimento de culpa, impulsos suicidas, abandono, baixa autoestima, medo da morte, hostilidade e raiva, desespero/desamparo. Ademais, sentimentos ruins e mágoa relacionados ao marido, falta de apetite sexual, incapacidade de se perdoar, pesadelos e tonturas dentre outros (SANTOS HCS, et al., 2017).

O abortamento autoprovocado gera por até 5 anos ansiedade, sentimento de culpa depressão, raiva e vergonha. Já os abortos espontâneos ocasionam alterações psicológicas até 6 meses e os sintomas são mais brandos em relação ao provocado (DOMINGUES RMSM, et al., 2020).

Um dos efeitos genuínos do abortamento e que geralmente aparecem anos depois é a depressão, que necessita de prevenção e tratamento e depende diretamente da causa e situações envolvidas no processo. Além de ser uma doença oculta e ignorada. A lembrança e os sentimentos de culpa não resolvidos transformam-se em gatilhos que podem surgir anos depois de uma forma inusitada. Essas emoções necessitam de atenção e são observadas na maioria das vezes através de perturbações emocionais ou de comportamento (LAINSCEK FGT, et al., 2019). 
Houve uma alteração nas manobras abortivas praticadas pelas mulheres com o avanço dos anos e da tecnologia. Ao invés de chás, folhas, uso de esponjas vaginais, permanganato de potássio e substancias corrosivas e materiais perfurocortantes. Os riscos físicos estão diretamente relacionados com a maneira de execução. Nos estudos científicos existem poucos dados que correlacionem tal ligação, entretanto em pesquisas fora do Brasil mostram que há uma diminuição do abortamento quando existe uma legislação mais branda (LOPES SDN e OLIVEIRA MHB, 2020).

A qualidade do primeiro atendimento é importante para prevenir deteriorização do estado clínico em relação ao abortamento inseguro. Em nações em desenvolvimento, ainda se valoriza a curetagem, porém já existem medicamentos e aspiração intrauterina que demonstram melhor prognóstico. Observa-se, também, que a falta de preparação da equipe para prestar atendimento e o baixo investimento em saúde pioram o atendimento prestado (MENEZES G, et al., 2020).

$\mathrm{Na}$ etapa inicial do fluxo de atenção um acolhimento respeitoso e humanizado é importante para garantia dos direitos humanos. Nessa etapa a escuta privilegiada e o tratamento digno podem ter um espaço importante para auxiliar no processo de acolhida à mulher e entender sobre o método abortivo utilizado para prevenir sequelas físicas e psicológicas futuras (CARDOSO VB, et al., 2021).

\section{CONSIDERAÇÕES FINAIS}

O aborto ocorre tanto em países desenvolvidos quanto em países em desenvolvimento, sendo o primeiro a maioria legalizado. Além disso, essa prática traz diversas consequências físicas, como por exemplo, perfuração do útero, restos de placenta pode ficar e levar a infecção e posteriormente até mesmo a infertilidade. Ademais, também pode ocorrer as consequências psicológicas, como a Síndrome Pós-aborto, em que a mulher passa a ter sintomas psicológicos, como tristeza, choro fácil, angustia, depressão e até autopunição, principalmente se realizado de forma insegura. No Brasil, a ilegalidade não impede que o aborto ocorra, estando principalmente relacionado à desigualdade social e afirmando-o como um problema de saúde pública de ordem global. Diante disso, dada à importância do assunto, torna-se necessário o desenvolvimento de mais pesquisas no âmbito penal e na saúde sobre este tema.

\section{REFERÊNCIAS}

1. AGUIAR BHK, et al. A legislação sobre o aborto nos países da América Latina: uma revisão narrativa. Comunicação Ciências Saúde, 2018; 29(1): 36-44.

2. AIRES FD, CARVALHO GM. O termo inicial da proteção da vida humana dependente no delito de aborto. Revista Quaestio luris, 2019; 12(3): 253-277.

3. BANDEIRA JC. Turismo de aborto: direitos reprodutivos e deslocamentos que visam a interrupção voluntária da gravidez. Frontera Norte, 2018; 30(59): 1-74

4. BELIN LL, RIZZOTTO CC. Menos Estigma, Pouco Aprofundamento: Uma Análise de Enquadramento Noticioso sobre o Direito ao Aborto. Revista Eco-Pós, 2021; 24(1): 159-187.

5. CARDOSO BB, et al. Aborto no Brasil: o que dizem os dados oficiais?. Cadernos de Saúde Pública, 2020; 36(1): e00188718.

6. CARDOSO VB, et al. Humanização na assistência de enfermagem à mulher em situação pós abortamento. Revista de Enfermagem - UFPE online, 2021; 15(1): 1-21.

7. CISNE M, et al. Aborto inseguro: um retrato patriarcal e racializado da pobreza das mulheres. Revista Katálysis, 2018; 21(3): 452-470.

8. CORREIA LL, et al. Tendência de abortos espontâneos e induzidos na região semiárida do Nordeste do Brasil: uma série transversal. Revista Brasileira de Saúde Materno Infantil, 2018; 18(1): 123-132.

9. CORRÊA MSM, et al. Acolhimento no cuidado à saúde da mulher no puerpério. Cadernos de saúde pública, 2017; 33(3): e00136215.

10.DOMINGUES RMSM, et al. Aborto inseguro no Brasil: revisão sistemática da produção científica, 20082018. Cadernos de Saúde Pública, 2020; 36(1): e00190418.

11.ESSY DB, JUSTO FS. Aborto legal no Brasil e as limitações ao exercício da autonomia das mulheres. Captura Críptica: direito, política, atualidade, 2019; 8(1): 5-19.

12.ESTEVES MF, et al. O uso inadequado do misoprostol como abortivo: uma revisão integrativa. Colloquium Vitae, 2021; 13(1): 22-34. 
13.FERRARI W, PERES S. Itinerários de solidão: aborto clandestino de adolescentes de uma favela da Zona Sul do Rio de Janeiro, Brasil. Cadernos de Saúde Pública, 2020; 36(1): e00198318.

14.FONSECA SC, et al. Aborto legal no Brasil: revisão sistemática da produção científica, 2008-2018. Cadernos de Saúde Pública, 2020; 36: e00189718.

15.GALLI B. Desafios e oportunidades para o acesso ao aborto legal e seguro na América Latina a partir dos cenários do Brasil, da Argentina e do Uruguai. Cadernos de Saúde Pública, 2020; 6(1): e00168419.

16. LACERDA E, et al. Reflexões sobre abortamento no brasil: um passeio na literatura. American Journal of Social Research, 2018; 1(5): 1-2.

17.LAINSCEK FGT, et al. Adolescente: aspectos emocionais frente ao aborto. Revista Cereus, 2019; 11(4): 72-83.

18.LOPES SDN, OLIVEIRA MHB. Meu corpo, minhas regras: mulheres na luta pelo acesso ao serviço público de saúde para a realização do aborto seguro. Saúde em Debate, 2020; 43(4): 20-33.

19.MAIA MN. Oferta de aborto legal na atenção primária à saúde. Revista Brasileira de Medicina de Família e Comunidade, 2021; 16(43): 1-8.

20.MARVÁN ML, et al. Atitudes perante a interrupção voluntária da gravidez em jovens mexicanos, e a opinião deles sobre o aborto inseguro como um problema de saúde pública. Cadernos de Saúde Pública, 2018; 34(10): e00192717.

21.MELO BC. Constitucionalização do aborto no âmbito do direito comparado. Caderno Virtual, 2019; 2(44): $1-15$.

22. MENEZES G, et al. Aborto e saúde no Brasil: desafios para a pesquisa sobre o tema em um contexto de ilegalidade. Cadernos de Saúde Pública, 2020; 36(1): e00197918.

23.PAES SC, et al. Aborto inseguro no Estado do Rio de Janeiro, Brasil: magnitude e evolução de 2008 a 2017. Cadernos de Saúde Pública, 2021; 37(10): 1-12.

24.POLETTO L, FERREIRA JBS. Reflexões acerca do aborto associado ao estupro. Revista Gestão e Tecnologia, 2020; 2(31): 56-65.

25.RODRÍGUEZ-RUÍZ AF, et al. Vantagens do bloqueio paracervical na aspiração manual a vácuo de pacientes com sangramento uterino anormal devido à hipertrofia endometrial e aborto incompleto no primeiro trimestre. Ginecologia e Obstetrícia do México, 2021; 89(4): 279-285.

26. RUIBAL A. A controvérsia constitucional do aborto no Brasil: Inovação na interação entre movimento social e Supremo Tribunal Federal. Revista Direito e Práxis, 2020; 11(2): 1166-1187.

27.SANTOS HCS, et al. Associação da idade e tempo de internação com tipo de parto e abortos. Revista Saúde.Com; 2017; 13(4): 1022-1026.

28.SANTOS KSS, et al. A experiência de mulheres no pós-parto sobre o planejamento familiar. Saúde e Pesquisa, 2019; 12(1): 177-185.

29.SOUZA NF, et al. Avanços e retrocessos da legislação brasileira relacionada a descriminalização do aborto. Revista da Mostra de Iniciação Científica e Extensão, 2020; 6(1): 1-5.

30.VALENZUELA MT, et al. Aborto, é um problema de saúde pública no Chile no campo da saúde maternoperinatal?. Revista médica de Chile, 2017; 145(8): 1013-1020. 